\title{
Convolutional Neural Networks In Medical Image Understanding
}

\author{
Megha Upreti ${ }^{1}$, Chitra Pandey ${ }^{2}$, Ankur Singh Bist ${ }^{3}$, Buphest Rawat ${ }^{4}$, Marviola Hardini ${ }^{5}$ \\ Graphic Era Hill University,Bhimtal, India ${ }^{1,2,3,4}$ \\ University of Raharja, JI. Jendral Sudirman No.40 Modernland, Cikokol, Tangerang ${ }^{5}$ \\ e-mail: mupreti@gehu.ac.in'1, cpandey@gehu.ac.in², ankur1990bist@gmail.com ${ }^{3}$, \\ bhr222@.gmail.com ${ }^{-4}$, marviola@raharia.info $^{5}$
}

Upreti, M., Pandey, C., Bist, A. S., Rawat, B., \& Hardini, M. (2021). Convolutional Neural Networks in Medical Image Understanding. Aptisi Transactions on Technopreneurship (ATT), 3(2), 6-12.

DOI: https://doi.org/10.34306/att.v3i2.188

\begin{abstract}
In the era of social media images/pictures play a vital role. Facebook, whatsapp, instagram everywhere we see a lot of pictures nowadays. Along with social media, the pictures play a very important role in medical science. Medical Image can help in diagnosis, clinical treatment and teaching tasks. Traditional classification of images has reached an end because of its time taking nature and efforts made to extract, select and classify. This problem is solved with the help of CNN(Convolutional neural network).In medical science we have treatment for body anomalies that were not there before. Using the deep learning models of CNN we can detect the disease like Cancer, Lung Infection and treat it. This article aims to provide a comprehensive survey of applications of CNNs in medical image understanding.
\end{abstract}

Keywords: Feature extraction, CNN, Muli-layer Neural Network, Medical data analysis.

\section{Introduction}

Any medical illness anomalies can be cured and reduced through timely diagnosis .For diagnosis we need to know the exact reason for the Anomaly. In Medical Science anomalies include glaucoma, diabetic retinopathy, tumors, interstitial lung diseases, heart diseases and tuberculosis. Diagnosis of affected areas can be done only after studying the Image taken by X-Ray, magnetic resonance imaging (MRI), computed tomography (CT), positron emission tomography (PET), single photon emission computed tomography or ultrasound scanning[1-6]. Through Medical Image Processing it becomes easy to detect the type of anomaly, size and the area to be treated. Due to less availability of human experts, their high charges and the rough estimate of the anomalies make it difficult to diagnose. Therefore there is a need for an expert system to detect the anomaly. Image understanding systems that use Machine Learning Techniques are fast evolving. One such intelligent and successful model is the Convolutional neural network (CNN) model, which automatically learns the needed features and extracts it for medical image understanding. CNN models have filters which are needed to extract and learn the features of the image for further understanding. The CNN model has a low error rate. Google net uses CNN to detect cancer with an accuracy of $89 \%$ whereas a human pathologist can achieve the accuracy of only $70 \%$.

\section{Deep Learning in Medical Domain}


CNN has become the choice for Medical Image Understanding and further helping to know the exact reason of an anomaly and treating. CNN is applied in various medical image understanding applications like cancer, tumor, skin Lesion, anomalies of heart eyes etc. CNN is also used in COVID-19 detection using chest X-Ray/CT Scan .A lot of research is going on in this field and various surveys have been published on this field. The objective of this survey is to offer a comprehensive overview of applications and methodology of CNNs.

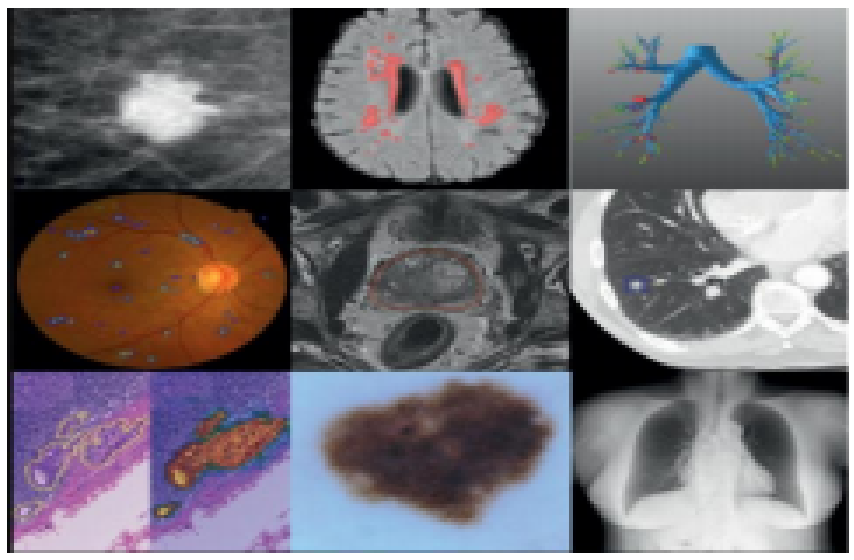

Figure1. Diseases tackled by Al

\subsection{Contributions and the Structure}

Primary contributions of this article are as follows:

- To briefly introduce medical image understanding and CNN.

- To convey that CNN has percolated in the field of medical image understanding.

- To identify the various challenges in medical image understanding.

- To highlight contributions of CNN to overcome those challenge

\subsection{Medical Image Understanding Important Task}
a. Medical image classification
b. Medical image segmentation
c. Medical image detection
d. Medical image localization

\subsection{Medical Image Classification}

Image classification refers to the task of extracting information. Medical image classification is a key technique of computer aided diagnosis (CAD) systems. Medical image classification is one of the most important problems in image recognition. The aim of image classification is to classify medical images into different categories to help doctors in disease diagnosis or further research.

Two steps of image classification is:

- Extracting features

- Build models that classify the image dataset

\section{Convolutional Layer}

\subsection{Medical Image Segmentation}

Segmentation is one of the most important and popular tasks. Segmentation refers to the task of partitioning a digital image into multiple segments. It divides an image into areas based on a specified description such as segmenting body organs/tissues in the medical application for border detection, tumor detection/segmentation and mass detection. The prior goal of the segmentation is to make things simpler and transform the representation of medical images into a meaningful subject.

\subsection{Medical Image Localization}


Image localization plays an important role in much medical image application. Localization means find where the object is and draw a bounding box around it.In image localization the goal is to be able to determine the location of a certain object in an image.

\subsection{Medical Image Detection}

Image detection is a technology that processes the image and detects objects in it, so medical image detection is the task of identifying medical-based objects within an image. This helps in determining the exact location of different organs and their orientation

\subsection{A Brief Introduction to CNNs}

Convolutional neural networks are designed to process data through multiple layers of arrays. Due to their plug-and-play architecture CNNs have achieved great success in image understanding. The introduction of convolutional neural network enabled a way to discover features in multi

dimensional data efficiently. Instead of using each pixel as a serrate input feature the Convolutional neurons are responsible for convolving a spatial region of the image.CNN represent the quantum leap in the field of image understanding [1] [2] [3] [4].

\subsection{CNN Architecture}

There are different types of layers that make up the architecture of a CNN. Convolutional layers, activation functions, pulling and fully-connected layers are the core building blocks of CNN. A sequence of these layers is often repeated multiple times and followed by fully connected layers preceding the output layer. In this section Convolutional layer, pooling layer and fully connected layers are introduced.Each of these layers has different parameters that can be optimized and performs a different task in input data.

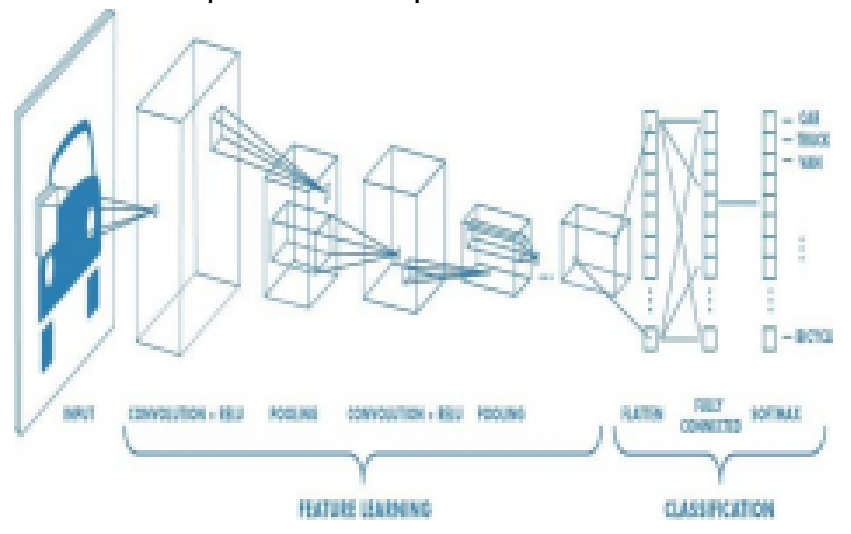

Figure2. Basic CNN architecture

The role of convolutional layer is to apply filters to the raw data in order to detect features and produce feature map.The objective of convolutional layer is to extract features, such as edges, colors, texture and gradient orientation. Convolutional layers are the layers where filters are applied to the original image, where most of the user-specified parameters are in the network. The most important parameters are the number of Kernels and the size of the Kernel. The output of the Convolutional layer is fed into an activation function layer.

\subsection{Activation Function}

Activation function is a very important feature. It is used to ensure that the representation in the input space is mapped to a different output space as per the requirement. Advantage of activation function-Differential is possible in all the activation functions and Stacking of network is possible, which helps us in creating the deep neural nets. The different activation functions.

\subsection{Sigmoid}

The term "sigmoid" means S-shaped, and it is also known as the squashing function. It takes a real- valued number $x$ and squashes it into range between 0 and 1 . This function has two simple properties-it can be used to model a conditional probability distribution and its derivative has a simple form.

\subsection{Tan Hyperbolic}


Tan hyperbolic or hyperbolic tangent function could be used as activation function.It takes a real valued number $x$ and squashes it between -1 to 1 .

3.10 Rectified Linear Unit (ReLU)

A Rectified Linear Unit is a form of activation function used commonly in deep learning models. The main advantage of using ReLU function over other activation functions is that it does not activate all the neurons at the same time. This nonlinear function takes a real valued number $x$ and converts $x$ to 0 if $x$ is negative. ReLU is the most often used for $\mathrm{CNN}$, takes less computation time and hence faster compared to the other two and it is expressed as $f(x)=\max (0, x)$.

\subsection{Pooling}

Pooling layer would reduce the number of parameters when the data is too large. reduce the dimensionality of each map but retain important information. It reduces the spatial size by aggregating data over space or feature type, controls over fitting and overcomes translation and rotational variance of images. Spatial pooling can be of different type- Max pooling, Average pooling, sum pooling.

\subsection{Fully Connected (FC) Layer}

Fully Connected Layer is simply, feed forward neural networks where all the inputs from one layer are connected to every activation unit of the next layer from all the inputs and all the connections have weights associated with them.

\subsection{CNN in Medical Image Understanding}

The basic CNN is made up of filters whose primary goal is to extract features of the image and learn it for further understanding.

\subsection{Breast Tumor}

Nowadays Breast Tumors are common in women. Breast Tumors can be detected by mammography. A mammogram is 3 an X-ray picture of the breast. Doctors use a mammogram to CNN.

\subsection{Heart Disease}

For Heart Disease detection ECG (Electrocardiogram) is used. An electrocardiogram is a simple, painless test that measures your heart's electrical activity. It's also known as an ECG or EKG. Every heartbeat is triggered by an electrical signal that starts at the top of your heart and travels to the bottom. Heart problems often affect the electrical activity of your heart. In other words An electrocardiogram (ECG) is a simple test that can be used to check your heart's rhythm and electrical activity. Sensors attached to the skin are used to detect the electrical signals produced by your heart each time it beats [5] [6] [7] [8] [9].

\subsection{Brain Tumors}

Brain Tumors can be detected from MRI. An MRI scan uses a large magnet, radio waves, and a computer to create a detailed, cross-sectional image of internal organs and structures. The scanner itself typically resembles a large tube with a table in the middle, allowing the patient to slide in. MRI can be used to detect brain tumors, traumatic brain injury, developmental anomalies, multiple sclerosis, stroke, dementia, infection, and the causes of headache [10] [11].

\subsection{COVID-19 Coronavirus Disease}

COVID-19 is a disease caused by a new strain of coronavirus. 'CO' stands for corona, ' $\mathrm{VI}$ ' for virus, and ' $\mathrm{D}$ ' for disease. Formerly, this disease was referred to as '2019 novel coronavirus' or '2019-nCoV.'.It is a global pandemic When the COVID-19 pandemic began, reverse-transcriptase PCR (RT PCR) tests were the first to be developed and widely deployed, and remain the primary tool used for diagnosis. While antigen tests are also being used and developed, they are not yet widely available and currently have variable reliability [12] [13]. Thus Chest X-Ray is used for the screening of COVID-19. Like other pneumonias, covid-19 pneumonia causes the density of the lungs to increase. This may be seen as whiteness in the lungs on radiography which, depending on the severity of the pneumonia. 


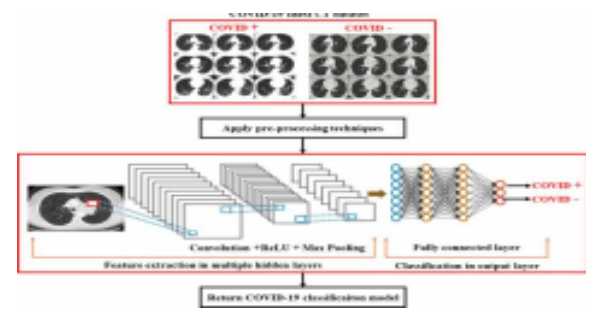

Figure3. COVID-19 detection using deep learning [14

CNN is applied in various images, there are various CNN models so it's difficult to select the best model for a particular problem. The challenge of CNN in medical imaging is the class imbalance problem in which the positive class is under-represented and the normal class represents the images. Designing CNN models for imbalance data is a challenging task. Researchers have dealt with this problem by applying augmentation of the underrepresented data [15] [16] [17].

CNNs' significant depth and enormous size require huge memory and higher computational resources for training. Medical image Understanding would be more efficient in the presence of background context or knowledge about the image to be understood. CNNs would be more efficient if the data consists of not only images, but also patient history.CNN has been successfully applied to various tasks in the medical image analysis domain by providing promising results in almost every case [18] [19].

Deep learning includes methods like CNN, recurrent neural networks. These topics are the area of research and a lot of research is going on in those areas. In this article all those aspects of image understanding cannot be covered since it is an ocean and the focus in the paper is only on a few important techniques involved [20] [21] [22].

\section{Conclusion And Future Work}

CNNs are used for image classification and recognition because of their high accuracy. The CNN follows a hierarchical model which works on building a network, like a funnel, and finally gives out a fully connected layer where all the neurons are connected to each other and the output is processed. Another reason why CNN are hugely popular is because of their architecture - the best thing is there is no need for feature extraction. The system learns to do feature extraction and the core concept of CNN is, it uses convolution of image and filters to generate invariant features which are passed onto the next layer. The main advantage of CNN compared to its predecessors is that it automatically detects the important features without any human supervision. For example, given many pictures of cats and dogs it learns distinctive features for each class by itself. CNN is also computationally efficient.

Performance improvement with model optimization 1. Fine tuning the model with subset data > Dropping few data samples for some of the overly sampled data classes. 2 Class weights $>>$ Used to train highly imbalanced (biased) databases, class weights will give equal importance to all the classes during training [23] [24] [25] [26].

\section{References}

[1] X. Zhou, Y. Li, and W. Liang, "CNN-RNN based intelligent recommendation for online medical pre-diagnosis support," IEEE/ACM Trans. Comput. Biol. Bioinforma., 2020.

[2] L. Liu, F.-X. Wu, Y.-P. Wang, and J. Wang, "Multi-Receptive-Field CNN for Semantic Segmentation of Medical Images," IEEE J. Biomed. Heal. Informatics, vol. 24, no. 11, pp. 3215-3225, 2020.

[3] M. Graziani, T. Lompech, H. Müller, A. Depeursinge, and V. Andrearczyk, "Interpretable CNN Pruning for Preserving Scale-Covariant Features in Medical Imaging," in Interpretable and Annotation-Efficient Learning for Medical Image Computing, Springer, 2020, pp. 23-32.

[4] P. Dutta, P. Upadhyay, M. De, and R. G. Khalkar, "Medical image analysis using deep convolutional neural networks: Cnn architectures and transfer learning," in 2020 International Conference on Inventive Computation Technologies (ICICT), 2020, pp. 
175-180.

[5] F. Ali et al., "A smart healthcare monitoring system for heart disease prediction based on ensemble deep learning and feature fusion," Inf. Fusion, vol. 63, pp. 208-222, 2020.

[6] R. Arnaout, L. Curran, Y. Zhao, J. Levine, E. Chinn, and A. Moon-Grady, "Expert-level prenatal detection of complex congenital heart disease from screening ultrasound using deep learning," medRxiv, 2020.

[7] T.-H. Nguyen, T.-N. Nguyen, and T.-T. Nguyen, "A deep learning framework for heart disease classification in an loTs-based system," in A Handbook of Internet of Things in Biomedical and Cyber Physical System, Springer, 2020, pp. 217-244.

[8] I. A. Alshawwa, H. Q. El-Mashharawi, M. Elkahlout, M. O. Al-Shawwa, and S. S. Abu-Naser, "Analyzing Types of Cherry Using Deep Learning," 2020.

[9] S. N. Pasha, D. Ramesh, S. Mohmmad, and A. Harshavardhan, "Cardiovascular disease prediction using deep learning techniques," in IOP Conference Series: Materials Science and Engineering, 2020, vol. 981, no. 2, p. 22006.

[10] T. Saba, A. S. Mohamed, M. El-Affendi, J. Amin, and M. Sharif, "Brain tumor detection using fusion of hand crafted and deep learning features," Cogn. Syst. Res., vol. 59, pp. 221-230, 2020.

[11] M. A. Khan et al., "Multimodal brain tumor classification using deep learning and robust feature selection: A machine learning application for radiologists," Diagnostics, vol. 10, no. 8 , p. $565,2020$.

[12] L. Cai, J. Gao, and D. Zhao, "A review of the application of deep learning in medical image classification and segmentation," Ann. Transl. Med., vol. 8, no. 11, 2020.

[13] E. K. Wang, C.-M. Chen, M. M. Hassan, and A. Almogren, "A deep learning based medical image segmentation technique in Internet-of-Medical-Things domain," Futur. Gener. Comput. Syst., vol. 108, pp. 135-144, 2020.

[14] D. Singh, V. Kumar, and M. Kaur, "Classification of COVID-19 patients from chest CT images using multi-objective differential evolution-based convolutional neural networks," Eur. J. Clin. Microbiol. Infect. Dis., vol. 39, no. 7, pp. 1379-1389, 2020.

[15] A. Vakanski, M. Xian, and P. E. Freer, "Attention-enriched deep learning model for breast tumor segmentation in ultrasound images," Ultrasound Med. Biol., vol. 46, no. 10, pp. 2819-2833, 2020.

[16] Y. Lei et al., "Deep learning-based breast tumor detection and segmentation in 3D ultrasound image," in Medical Imaging 2020: Ultrasonic Imaging and Tomography, 2020, vol. 11319, p. 113190Y.

[17] Y. Jiménez-Gaona, M. J. Rodríguez-Álvarez, and V. Lakshminarayanan, "Deep-Learning-Based Computer-Aided Systems for Breast Cancer Imaging: A Critical Review," Appl. Sci., vol. 10, no. 22, p. 8298, 2020.

[18] A. S. Assiri, S. Nazir, and S. A. Velastin, "Breast tumor classification using an ensemble machine learning method," J. Imaging, vol. 6, no. 6, p. 39, 2020.

[19] Y. Jiang, M. Yang, S. Wang, X. Li, and Y. Sun, "Emerging role of deep learning-based artificial intelligence in tumor pathology," Cancer Commun., vol. 40, no. 4, pp. 154-166, 2020.

[20] K. Dev, S. A. Khowaja, A. S. Bist, V. Saini, and S. Bhatia, "Triage of potential COVID-19 patients from chest X-ray images using hierarchical convolutional networks," Neural Comput. Appl., pp. 1-16, 2021.

[21] K. Arora, A. S. Bist, S. Chaurasia, and R. Prakash, "Analysis of deep learning techniques for COVID-19 detection," Int J Sci Res Eng Manag i, vol. 4, no. 4, pp. 1-5, 2020.

[22] S. Wang et al., "A fully automatic deep learning system for COVID-19 diagnostic and prognostic analysis," Eur. Respir. J., vol. 56, no. 2, 2020.

[23] A. Esteva et al., "Deep learning-enabled medical computer vision," NPJ Digit. Med., vol. 4, no. 1, pp. 1-9, 2021.

[24] S. Bhattacharya et al., "Deep learning and medical image processing for coronavirus (COVID-19) pandemic: A survey," Sustain. cities Soc., vol. 65, p. 102589, 2021. 
[25] L. Zhang et al., "Generalizing deep learning for medical image segmentation to unseen domains via deep stacked transformation," IEEE Trans. Med. Imaging, vol. 39, no. 7, pp. 2531-2540, 2020.

[26] A. Smailagic et al., "O-MedAL: Online active deep learning for medical image analysis," Wiley Interdiscip. Rev. Data Min. Knowl. Discov., vol. 10, no. 4, p. e1353, 2020. 